\section{Verbesserte Asthmakontrolle im Blick}

\begin{abstract}
A uf ein Phänomen machte Prof. Dr. Malcom Sears aus Ontario, Kanada, aufmerksam: Jedes Jahr Anfang September zeigt sich bei Kindern und Jugendlichen in Kanada, aber auch in einigen europäischen Ländern ein Maximum bei den stationären Aufnahmen wegen Asthma bronchiale. Der Grund dafür ist vermutlich der Schulanfang mit einsetzender psychischer Belastung, neuem Kontakt zu Personen mit viralen Infektionen und Exposition gegenüber einem anderen Allergenspektrum. Nicht zuletzt sind die betroffenen Kinder, wie Erhebungen gezeigt haben, auch medikamentös deutlich schlechter eingestellt.

Aktuell gibt es sechs verschiedene Leitlinien zur Therapie des Asthma bronchiale. Dabei ist ein klarer Paradigmenwechsel zu erkennen, der von der starren, stufenorientierten Behandlung in ein flexibleres System überführt, bei dem die Asthmakontrolle unter der jeweiligen Medikation im Vordergrund steht. Prof. Dr. Jürgen Seidenberg aus Oldenburg wies auf ein 2008 in der Zeitschrift Thorax publiziertes Review von Joos et al.
\end{abstract}

hin, in dem die Kombinationen Salmeterol plus inhalative Glukokortikoide und Montelukast plus inhalative Glukokortikoide miteinander verglichen wurden. In Kurzzeitstudien bis zu zwölf Wochen zeigte sich die Kombination mit dem $\beta$ Mimetikum zunächst überlegen, allerdings glich sich dieser Wirkunterschied in Langzeitstudien von 48 Wochen wieder aus. Es sollten auch Sicherheitsas-

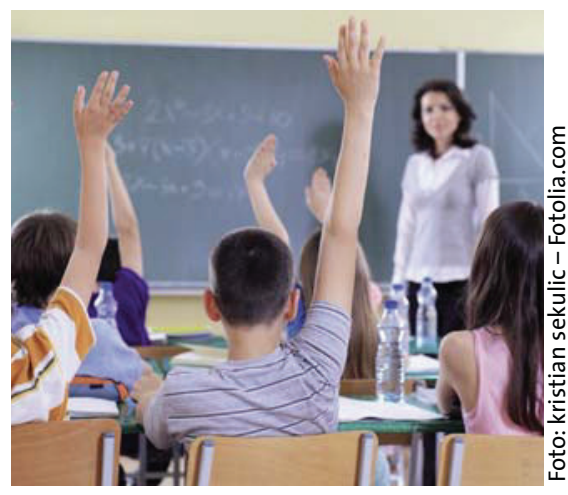

Der Schulanfang im September markiert auch ein Maximum in der Zahl der stationären Aufnahmen wegen Asthmaexazerbationen. pekte im Vordergrund stehen, die bei unerwarteten schweren Nebenwirkungen durch Salmeterol zugunsten von Montelukast sprechen könnten.

Prof. Dr. J. Christian Virchow, Rostock, stellte Daten der MONICA-Studie vor. In dieser offenen Studie erhielten 1.681 Patienten, die mit leichtem bis mittelgradigem Asthma unter inhalativen Glukokortikoiden bzw. inhalativen Glukokortikoiden und lang wirksamen $\beta$-Mimetika nicht zufriedenstellend eingestellt waren, zusätzlich Montelukast. Rund $80 \%$ der Patienten zeigten im Asthmakontrolltest (ACT) eine Besserung des Asthmas durch die Behandlung mit dem Leukotrienantagonisten. Asthmakranke, die nur inhalative Glukokortikoide als Vorbehandlung angewandt hatten, Patienten mit niedrigen Werten im ACT bei der Eingangsuntersuchung sowie Patienten mit allergischer Rhinitis profitierten besonders von der Therapie mit Montelukast (Singulair ${ }^{\circledR}$ )

Industriesymposium „Asthma im Kindesund Erwachsenenalter - alles unter Kontrolle?". 4. Gemeinsamer Deutscher Allergiekongress, Berlin, 3.-6. September 2009. Veranstalter: MSD, Haar

\title{
Antihistaminikum mit Zusatzeffekt
}

D as Antihistaminikum Rupatadin blockiert neben dem H1-Rezeptor zusätzlich den Rezeptor des sekundären Mastzellmediators PAF (,platelet-activating factor"). Dieser duale Ansatz bewährt sich. Bereits in vier Phase-IIIStudien konnte die Wirksamkeit von Rupatadin, Handelsname Rupafin ${ }^{\circledR}$, bei perennialer wie saisonaler allergischer Rhinitis belegt werden. Sowohl in der Dosierung $10 \mathrm{mg}$ als auch $20 \mathrm{mg}$ täglich reduzierte das nicht sedierende Antiallergikum die Symptome der Rhinitis signifikant besser als Plazebo.

In verschiedenen Vergleichsstudien war Rupatadin bei saisonaler Rhinitis mindestens so wirksam wie Cetirizin, Ebastin, Loratadin und Desloratadin, in einigen Endpunkten sogar überlegen, betonte Prof. Dr. Ralph Mösges, Köln. Eine aktuelle Phase-IV-Studie sollte die
Wirksamkeit über zwölf Wochen im Praxisalltag überprüfen. Die Untersucher verglichen plazebokontrolliert den Effekt von $10 \mathrm{mg}$ Rupatadin einmal täglich mit dem von Cetirizin $10 \mathrm{mg}$ einmal täglich bei 543 Patienten mit perennialer allergischer Rhinitis. Nach zwölf Wochen hatte Rupatadin den Gesamtsymptomscore signifikant gegenüber Plazebo gesenkt, nicht aber Cetirizin in der verwendeten Dosis.

Eine weitere Post-Marketing-Studie aus dem Jahr 2006 belegte die Langzeitsicherheit von Rupatadin über ein Jahr bei perennialer Rhinitis bei 315 Patienten. Nach vier Monaten vorhandene unerwünschte Wirkungen, wie trockener Mund (2,2\%) oder Kopfschmerzen (6,5\%), waren nach zwölf Monaten praktisch völlig verschwunden, 5,8\% der Patienten berichteten nach einem
Jahr noch von Somnolenz - letztlich aber alles Nebenwirkungsinzidenzen auf Plazeboniveau.

In einer weiteren Studie verbesserte Rupatadin die Lebensqualität von $92 \mathrm{~Pa}$ tienten mit perennialer allergischer Rhinitis über den Zeitraum von einem Jahr signifikant gegenüber Plazebo, berichtete Mösges. Insgesamt sieht der Kölner Allergologe einen klaren Vorteil für das verordnungsfähige Antihistaminikum mit PAF-Hemmung: „Im Effektmaß ist Rupatadin verschreibungsfreien Generika überlegen." Die Substanz hat ein breites Wirkspektrum und wirkt auch antiobstruktiv, was Mösges auf den kombinierten Wirkansatz zurückführt. $\quad f k$

Industriesymposium „Rupatadin - ein neues Antiallergikum mit dualem Wirkmechanismus". 4. Gemeinsamer Deutscher Allergiekongress, Berlin, 3.-6. September 2009. Veranstalter: MerckleRecordati, Ulm 